\title{
Independence and separability of volume and mass in the size-weight illusion
}

\author{
CRYSTAL D. OBERLE and ERIC L. AMAZEEN \\ Arizona State University, Tempe, Arizona
}

\begin{abstract}
Numerous size-weight illusion models were classifiedin the present article according to general recognition theory (Ashby \& Townsend, 1986), wherein the illusion results from a lack of perceptual separability, perceptual independence, decisional separability, or a combination of the three. These options were tested in two experiments in which a feature-complete factorial design and multidimensional signal detection analysis were used (Kadlec \& Townsend, 1992a, 1992b). With haptic touch alone, the illusion was associated with a lack of perceptual and decisional separability. When the participant viewed the stimulus in his or her hand, the illusion was associated only with a lack of decisional separability. Visual input appeared to improve the discrimination of mass, leaving only the response bias due to expectation.
\end{abstract}

In the study of weight perception, one phenomenon stands out in its importance: the size-weight illusion. First demonstrated by Charpentier (1891; see the discussion in Murray, Ellis, Bandomir, \& Ross, 1999) and studied extensively thereafter (see Jones, 1986, for a review), this illusion refers to the commonly observed inverse relationship between physical volume and the report of perceived heaviness; as physical volume increases, the magnitude of the perceptual reports for perceived heaviness decreases (e.g., Stevens \& Rubin, 1970). The illusion is important because it shows that reports of perceived heaviness are not a function of mass alone. Any valid model of weight perception, then, must identify how mass and volume are combined, either within the stimulus or through some cognitive process.

A size-weight illusion has been demonstrated with various modes of touch and visual input (see Gibson, 1966, and Loomis \& Lederman, 1986, for varieties of touch). The most common scenario has participants grasp and lift an object that is in clear view; yet the illusion has also been examined under purely haptic conditions in which participants may grasp but not view each stimulus (Ellis \& Lederman, 1993; Pick \& Pick, 1967). In each of these two studies, it was concluded that haptic information about volume produced a stronger effect than did visual information on perceived heaviness. In addition to its presence with haptic touch, the size-weight illusion has also been observed with dynamic, or kinesthetic, touch (Amazeen, 1997, 1999; Amazeen \& Turvey, 1996; Kloos \& Amazeen, 2002). Under these conditions, participants held visually occluded stimuli by a handle. Volume was available to the participant only through the muscular dynamics involved in holding and wielding the stimuli. When the dynamic touch illusion is compared with a condition in which the participants can see the stimulus that they are holding by

Correspondence concerning this article should be sent to C. D. Oberle, Department of Psychology, Box 871104, Arizona State University, Tempe, AZ 85287 (e-mail: crystal.oberle@asu.edu). a handle, a qualitative change in the illusion results, suggesting that vision makes additional information or processing available (Amazeen, 1997). In sum, although the illusion has been evident with a variety of modes of touch and visual input, the findings differ quantitatively and qualitatively, suggesting that illusion models must be sensitive to the modality of perception being used.

Many models suggest that the illusion is due to perceptpercept coupling (see Jones, 1986, for a review). According to such models, it is an interaction, or apparent lack of independence, between the perceptual effects of mass and volume that leads to the observed effect of physical volume on the participant's report of perceived heaviness. Signal detection theory (e.g., Green \& Swets, 1966), however, identifies other processes that occur in making a perceptual report. The simplest form of a signal detection model assumes variation across encounters with a stimulus, resulting in a distribution of perceptual effects. Once a stimulus property gives rise to a perceptual effect, a decision rule is applied to that effect, and then a report is given that is based on the combination of the decision rule and the perceptual effect. Percept-percept coupling refers only to an interaction between the perceptual effects associated with mass and volume. It is possible, though, that a stimulusresponse covariation such as that associated with the sizeweight illusion could result from an interaction in any one of these processes. In the present experiments, a multidimensional signal detection paradigm was adopted to evaluate the potential sources of the stimulus-response covariation that is typical of the size-weight illusion for haptic touch with and without vision.

\section{Sources of Covariation}

Ashby and Townsend's (1986) general recognition theory (GRT) extends signal detection theory to address the perceptual processing of multiattribute stimuli (e.g., objects varying in volume and mass; other multidimensional generalizations of signal detection theory have been offered 
by Macmillan \& Creelman, 1991, and Wickens \& Olzak, 1992). The purpose for this extension was to identify the varieties of perceptual independence - that is, the potential sources of an interaction across perceptual dimensions, such as that associated with the size-weight illusion. The three potential sources of a stimulus-response covariation at the psychological space level concern perceptual separability, perceptual independence, and decisional separability.

Perceptual separability. Perceptual separability refers to the influence of the physical dimensions on the perceptual effects. Two physical dimensions are perceptually separable if the perceptual effect on one dimension is unaffected by the physical level (as opposed to the perception) of the other dimension of a multiattribute stimulus (Ashby \& Townsend, 1986). For example, volume and mass would be perceptually separable if perceived volume did not depend on the level of mass and if the perceived heaviness did not depend on the level of volume. ${ }^{1}$ Some models of the size-weight illusion, however, suggest that this illusion results from a lack of perceptual separability. Two such models are the density model (H. E. Ross, 1969; J. Ross \& Di Lollo, 1970) and the inertial model (Amazeen, 1997, 1999; Amazeen \& Turvey, 1996). In each of these models, perceived heaviness is considered to be a function of a stimulus property, either density or rotational inertia, that is itself a function of mass and volume.

Another way of portraying this lack of perceptual separability is with equal-probability contours from a multidimensional signal detection experiment on the illusion. This experiment would involve a feature-complete factorial design, also referred to as a complete identification paradigm, in which there is a factorial combination of two levels of both volume and mass and a perceptual report for each of these dimensions is given. With such a design, four three-dimensional perceptual distributions arise, one for each stimulus. The equal-probability contours are determined by cutting a plane perpendicular to the frequency axis. Hypothetical equal-probability contours are shown in the top of Figure 1 for models suggesting that the illusion is due to a lack of perceptual separability. In this figure, the contours corresponding to the large stimuli are shifted to the left, indicating that perceived heaviness is dependent on the level of physical volume and decreases in magnitude as volume increases. These illusion models, mentioned above, make no claims regarding the effects of mass on perceived volume, so the proposed lack of perceptual separability may be assumed to be unidirectional in that perceived volume does not depend on the level of mass.

Perceptual independence. Perceptual independence refers to the influence of one perceptual effect on another. Two perceptual effects are independent if one is unaffected by variations in the other (Ashby \& Townsend, 1986). A lack of perceptual independence is theoretically comparable to Epstein's (1982) percept-percept coupling construct. Many models of the size-weight illusion suggest that this illusion is due to an apparent lack of perceptual independence-namely, that there is an effect of per-

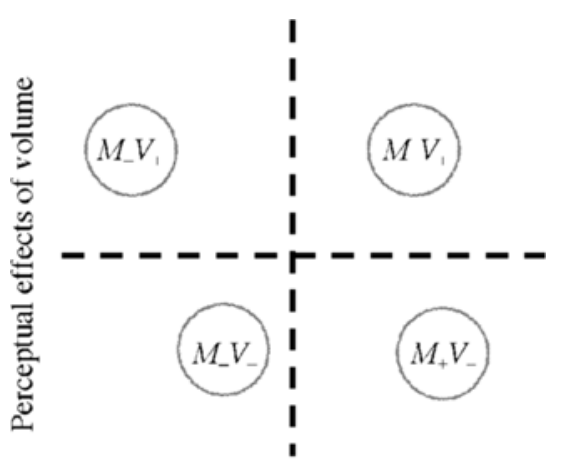

Perceptual effects of mass

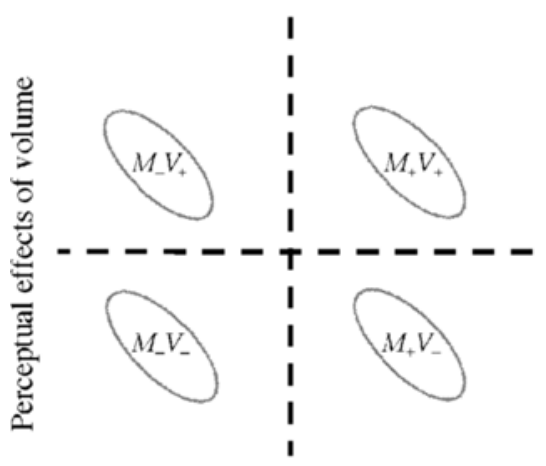

Perceptual effects of mass

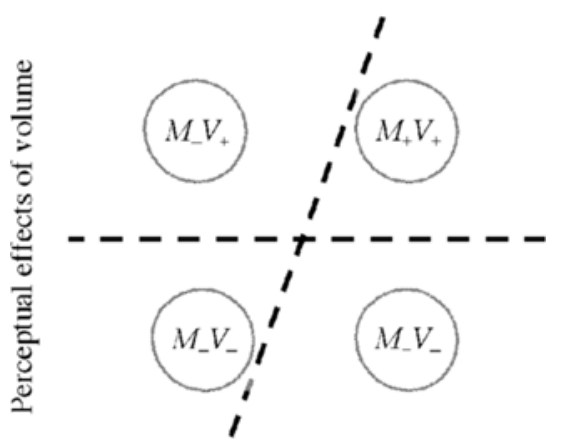

Perceptual effects of mass

Figure 1. Hypothetical equal-probability contours for models suggesting that the size-weight illusion is due to a lack of perceptual separability (top), a lack of perceptual independence (middle), and a lack of decisional separability (bottom). $M$ and $V$ represent the corresponding physical dimensions, and the subscripts refer to the levels of these physical dimensions (+ for the higher level, - for the lower level).

ceived volume on perceived heaviness that produces the observed covariation between physical volume and the participant's report of perceived heaviness. Hypothetical equal-probability contours are shown in the middle of Figure 1 for such models, which specifically suggest that perceived volume has an inverse effect on perceived heaviness. In this figure, the contours corresponding to each stimulus are ellipses slanted to the left, representing the 
negative correlation between the two perceptions and revealing the bidirectional nature of this percept-percept coupling.

Two models proposing a lack of perceptual independence are the information integration model (Anderson, 1970, 1972) and the expectation model (Davis \& Roberts, 1976; H. E. Ross \& Gregory, 1970). Although there are differences between them, each model assumes that an interaction between perceived heaviness and perceived volume occurs because of the positive correlation between volume and mass in nature. This correlation leads to an expectation of mass based on perceived volume. Because of this expectation, perceived heaviness is inversely influenced by the perceived volume. In sum, this expectation has an effect at the level of the perceptual effect. It should be noted, however, that signal detection theory identifies a role for expectations at the decisional level of perceptual processing. These models, then, combine features of a lack of both perceptual independence and decisional separability.

Decisional separability. The third variety of perceptual independence occurs at the decisional level of perceptual processing. Decisional separability refers to the influence of one dimension on the decision rule for the other (Ashby \& Townsend, 1986). Volume and mass would be decisionally separable if the decision criterion, used to transform perceived heaviness into the report of perceived heaviness, did not depend on the level of volume, and vice versa. It is possible, though, that systematic variations in the decision criterion applied to perceived heaviness as a function of volume could produce a size-weight effect. Hypothetical equal-probability contours are shown in the bottom of Figure 1 for models that may suggest that the size-weight illusion is due to a lack of decisional separability. In this figure, the decision criterion for perceived heaviness is slanted to the right, indicating that decisional bias depends on the level of physical volume and that people are biased to report that large objects feel light and small objects feel heavy. If no claims are made regarding the effects of mass on perceived volume, this lack of decisional separability may be assumed to be unidirectional in that placement of the decision criterion for perceived volume does not depend on the level of mass.

In contrast to perceptual independence, the effect identified by decisional separability is not on the perception itself. Rather, decisional separability refers to a bias in responding. This distinction is important when the information integration and the expectation models are evaluated, because they rely on a bias that is based on experience in the natural world. Although these particular models suggest that the bias influences the perceptual effect, it could just as well influence the decision criterion. In fact, signal detection theory often treats biases resulting from changes in expectations in terms of the decision criterion. It is important, therefore, to be able to distinguish among these potential sources of covariation within a data set.

Using a multidimensional signal detection analysis (MSDA; Kadlec, 1995, 1999a, 1999b; Kadlec \& Townsend, 1992a, 1992b; for other example applications, see also Kadlec \& Hicks, 1998; Thomas, 2001; Wenger \& Ingvalson, 2002), the experiments reported in the present article were performed to evaluate each of these sources of covariation from GRT (Ashby \& Townsend, 1986) in the sizeweight illusion. To date, an analysis of the sort used here has been applied to the size-weight illusion only in conditions of dynamic touch without visual input (Amazeen, 1999). The results of that analysis suggested that volume and mass were perceptually independent. To account for the presence of the size-weight illusion, it was concluded that perceived heaviness was a function of both volume and mass, perhaps due to the fact that they both factor into an object's rotational inertia, which is the stimulus property used by dynamic touch to perceive heaviness. This conclusion amounts to a lack of perceptual separability of volume and mass. This finding will be compared with the results of the present experiments, in which the potential sources of covariation were tested in a haptics-only condition and a haptics + vision condition. The goal is to determine which classes of models of the illusion would be appropriate for dynamic touch, haptic touch without visual input, and haptic touch with visual input.

\section{EXPERIMENT 1}

Experiment 1 tested for perceptual separability, perceptual independence, and decisional separability of volume and mass in a haptics-only condition, in which the participant was allowed to hold and manipulate the entire stimulus with his or her hand.

\section{Method}

\section{Participants}

Eleven female and 4 male Arizona State University undergraduate students participated in this experiment in partial fulfillment of an introductory psychology course requirement. Two participants were left-handed (based on self-reports of which hand they throw a ball with and write with), and the others were right-handed.

\section{Design}

A feature-complete factorial design was used, in which there was a factorial combination of mass and volume, as well as a perceptual report for each on every trial. There were two levels each of volume and mass. On each trial, the participant gave reports of perceived volume (whether the stimulus felt large or small) and perceived heaviness (whether the stimulus felt heavy or light). With this design, the changes in estimated response probabilities and signal detection parameters could be evaluated across variations in both volume and mass.

\section{Materials}

The stimuli consisted of four wooden cylinders. The large $\left(V_{+}\right)$ and small $\left(V_{-}\right)$levels of volume were $46.0 \mathrm{cc}$ (diameter $=3.1 \mathrm{~cm}$, height $=6.1 \mathrm{~cm})$ and $37.0 \mathrm{cc}($ diameter $=2.9 \mathrm{~cm}$, height $=5.6 \mathrm{~cm})$, respectively. ${ }^{2}$ The heavy $\left(M_{+}\right)$and light $\left(M_{-}\right)$levels of mass were 25.0 and $20.0 \mathrm{~g}$, respectively. Mass was varied independently of volume by drilling holes lengthwise in some of the cylinders. Care was taken to ensure that the drilled holes were distributed evenly throughout each cylinder. The ends of each cylinder were covered with balsa wood to provide a smooth uniform surface that would provide no clues about the manipulations of volume or mass. 


\section{Procedure}

The participant sat in a chair with his or her right side parallel to a curtain. The right arm extended around the curtain so that the right forearm rested on a table on the opposite side of the curtain, where the stimuli were presented. This conf iguration allowed for unobstructed holding, while eliminating all visual cues. Prior to the experimental trials, the participant was allowed to hold and manipulate each stimulus once to familiarize himself or herself with the stimuli. At no time was the participant allowed to view any of the stimuli. During the experimental trials, the stimulus was placed on the table behind the curtain, and the participant was instructed to lift the stimulus and to give reports of both perceived volume (whether he or she believed the stimulus was the large $\left[v_{+}\right]$or the small $\left[v_{-}\right]$one) and perceived heaviness (whether he or she believed it was the heavy $\left[m_{+}\right]$or the light $\left[m_{-}\right]$one). Each participant completed 400 randomized trials, 100 for each stimulus. No time constraints were used, and no feedback was given between trials.

\section{Analysis}

The MSDA developed by Kadlec and Townsend (Kadlec, 1995, 1999a, 1999b; Kadlec \& Townsend, 1992a, 1992b) was used. This analysis extends that of Ashby and Townsend (1986) by providing additional comparisons of estimated signal detection parameters. The entire analysis has two parts: the macroanalyses and the microanalyses. The macroanalyses (marginal response invariance and marginal signal detection analyses) involve the evaluation of responses on one dimension (e.g., $m$ ) across variations in the nontargeted stimulus dimension (e.g., $V$ ). The microanalyses (sampling independence and conditional signal detection analyses) involve the evaluation of responses on one dimension (e.g., $m$ ) across variations in the other perceptual report (e.g., $v$ ). As was discussed in the introduction, these analyses evaluate the effects of physical volume on perceived heaviness separately from the effects of perceived volume. The possible conclusions that may be drawn from these tests are illustrated in Figure 2.

Marginal response invariance. The first macroanalysis is the test of marginal response invariance (MRI). For this test, the proportions of correct responses on a given level of one dimension are compared across levels of the nontargeted stimulus dimension. For example, the proportion of heavy responses for the heavy and small stimulus, $p\left(m_{+} \mid M_{+} V_{-}\right)$, is compared with the proportion of heavy responses for the heavy and large stimulus, $p\left(m_{+} \mid M_{+} V_{+}\right)$. Each pair
Analyses

Conclusions

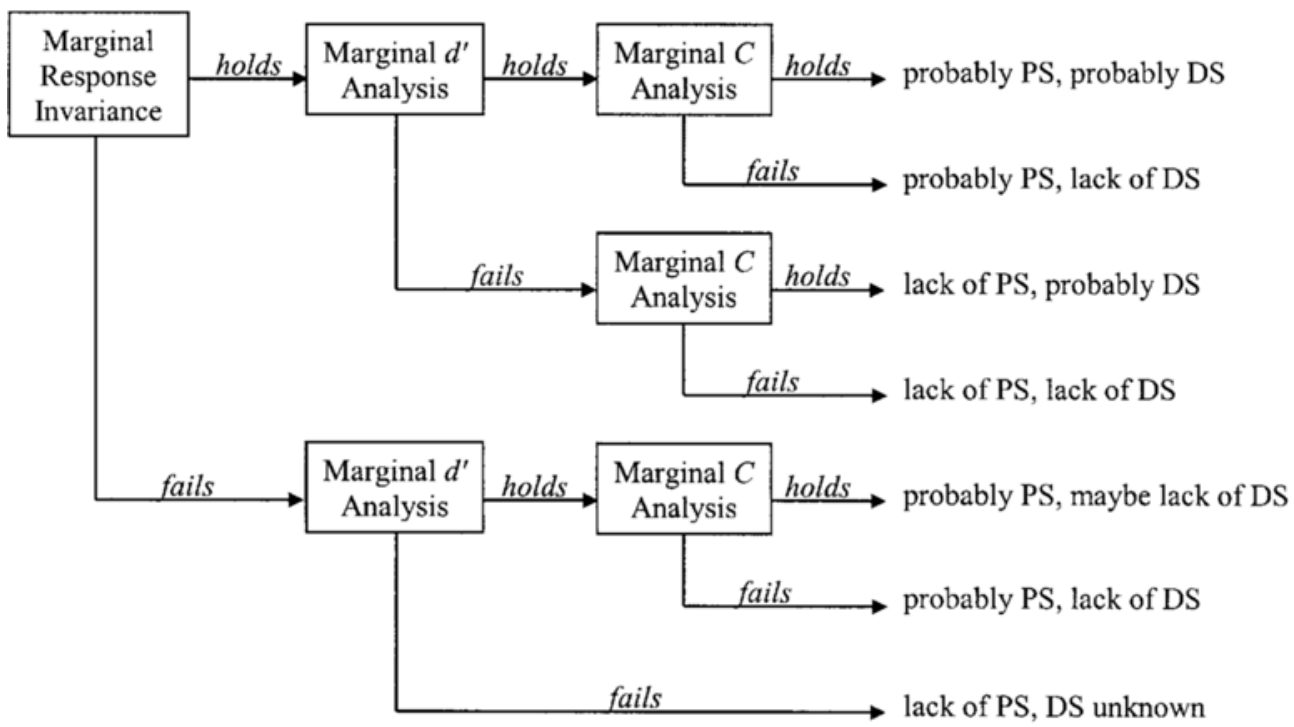

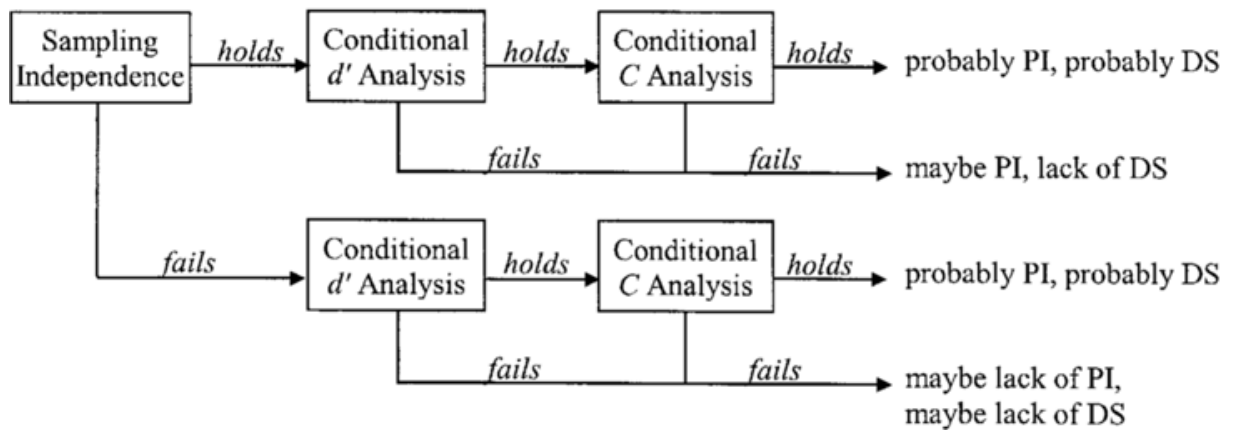

Figure 2. Flow charts representing the possible outcomes of the macroanalyses (top) and microanalyses (bottom), as well as the inferences that may be drawn from these outcomes regarding perceptual independence (PI), perceptual separability (PS), and decisional separability (DS). These illustrations are based on Tables 1 and 2 of Kadlec (1995; see also Kadlec, 1999a). 
of response proportions is compared using a binomial test. If these proportions are not equal, the nontargeted stimulus dimension is influencing the perceptual report.

Marginal signal detection analyses. The remaining macroanalyses evaluate estimates of discriminability $(d)$ and decisional bias $(C)$ from signal detection theory (e.g., Green \& Swets, 1966). Although the formal analyses of Kadlec and Townsend (1992a) relied on $\beta$ as a measure of decisional bias, $C$ may be used as the primary measure of decisional bias, due to the lack of a significance test for $\beta$ (Kadlec, 1995). These analyses compare estimates of $d$. and $C$ for a given report (e.g., $m$ ) across levels of the nontargeted stimulus dimension (e.g., at $V_{-}$and $V_{+}$). Each pair of $d$, and $C$ values is compared using a $z$ test based on estimates of the standard error associated with each signal detection parameter (Gourevitch \& Galanter, 1967; Kadlec, 1995, 1999a, 1999b; Macmillan \& Creelman, 1991; Marascuilo, 1970; Miller, 1996; Robertson \& Morgan, 1990). The results of these tests will indicate whether discriminability and decisional bias vary for each report as a function of the nontargeted stimulus dimension.

Inferences from macroanalyses. On the basis of the definitions of perceptual and decisional separability, if each is present, we should expect the marginal response probabilities and the marginal signal detection parameters to be invariant across levels of the nontargeted stimulus variable. Failures of these tests will allow us to make inferences about separability (see the top of Figure 2). Inferences about perceptual separability are made on the basis of marginal $d$. If discriminability varies, as would be suggested by unequal marginal $d$ s, there is a lack of perceptual separability. Inferences about decisional separability depend on the outcomes of both the MRI and the marginal signal detection analyses. When MRI holds, unequal marginal $C$ s (suggesting a shift in the criterion) imply a lack of decisional separability. When MRI fails, the test of marginal $d$. will determine whether the failure of MRI is likely a function of a lack of decisional separability. With equal marginal $d$, s, it is likely that the failure of MRI is related to a lack of decisional separability. This conclusion is strengthened when the marginal $C$ s are also not equal. When $d$ 's are not equal, however, it is impossible to determine whether the failure of MRI is related to decisional separability. Additional tests for decisional separability are offered by the microanalyses.

Sampling independence. The first microanalysis is the test of sampling independence. Sampling independence is a test of statistical independence conducted within a single stimulus in order to isolate the effects of variations in the perceptual effect from variations in the stimulus. For each stimulus (e.g., $M_{+} V_{+}$), the proportion of correct joint responses (e.g., $p\left(m_{+} v_{+} \mid M_{+} V_{+}\right)$is compared with the product of the proportion of correct responses on one dimension (e.g., $p\left(v_{+} \mid M_{+} V_{+}\right)$and the proportion of correct responses on the other dimension (e.g., $p\left(m_{+} \mid M_{+} V_{+}\right)$. Each pair of response proportions is compared using a binomial test. If these proportions are not equal, one report is not independent from the other.

Conditional signal detection analyses. The remaining microanalyses evaluate conditional estimates of discriminability $\left(d^{\prime}\right)$ and decisional bias $(C)$ from signal detection theory (e.g., Green \& Swets, 1966). These estimates are termed conditional because each estimate is conditioned on a particular response to the level of the nontargeted stimulus dimension. So, for a given level of the nontargeted stimulus dimension (e.g., $V_{+}$), the reports on one dimension (e.g., $m$ ) are compared across variations in reports on the nontargeted perceptual dimension (e.g., at $v_{-}$and $v_{+}$). The results of these tests will indicate whether discriminability and decisional bias vary for each report as a function of the other perceptual report.

Inferences from microanalyses. Inferences about perceptual independence and decisional separability may be drawn from these tests (see the bottom of Figure 2). Unless the pairs of both conditional signal detection parameters are equal, a failure of sampling independence indicates that the perceptual effects may not be independentthat there is an apparent lack of perceptual independence of volume and mass. If the pairs of either or both of the conditional signal detection parameters are unequal, decisional separability most likely does not exist.

\section{Results}

The response frequencies for all stimuli and all participants combined are presented in Table 1. Collapsing across levels of mass, as physical volume increased, the frequency of light responses increased (1,296 for the small stimuli and 1,892 for the large stimuli), and the frequency of heavy responses decreased (1,704 for the small stimuli and 1,108 for the large stimuli), revealing a size-weight illusion. Because heavy responses may be thought of as greater in magnitude than are light responses, these data are consistent with the results of non-GRT researchers (e.g., Stevens \& Rubin, 1970), who have shown that the magnitude of the perceptual reports for perceived heaviness decreases as physical volume increases.

For comparison purposes, analyses were conducted on the pooled data; in addition, the data were also analyzed on a participant-by-participant basis, another appropriate method of analysis for a large data set (Macmillan \& Creelman, 1991). Test failures were suggested when significant differences in values (e.g., $d$, for perceived heaviness across levels of volume) occurred for both types of analyses. The nonparametric sign test was used for these individual analyses to make inferences about the population. For these sign tests, all analyses were performed separately on each participant's data. Increases in values (e.g., increase in $d$ value for perceived heaviness from the small to the large stimuli) were assigned positive signs, whereas decreases in values were assigned negative signs. A binomial distribution table was then used to determine significance. An alpha level of .01 was used in all analyses, because doing multiple statistical tests on the same data set warrants decreasing the alpha level.

\section{Marginal Response Invariance}

The results from the pooled data for perceived heaviness reveal that, for both levels of mass, the proportion of light responses increased significantly as volume increased $(p<.001$; see Table 2). For each level of mass, 14 out of 15 participants followed this trend $(z=3.48, p<.001$, for each comparison). The results from the pooled data for perceived volume reveal that the proportion of small responses for the small stimuli increased significantly as

Table 1

Response Frequencies for Experiment 1

\begin{tabular}{lrrrr}
\hline Report & $M_{-} V_{-}$ & $M_{-} V_{+}$ & $M_{+} V_{-}$ & $M_{+} V_{+}$ \\
\hline$m_{-} v_{-}$ & 885 & 133 & 213 & 44 \\
$m_{-} v_{+}$ & 130 & 1,209 & 68 & 506 \\
$m_{+} v_{-}$ & 465 & 44 & 1,072 & 126 \\
$m_{+} v_{+}$ & 20 & 114 & 147 & 824 \\
\hline
\end{tabular}

Note- $M$, mass; $V$, volume; $m$, perceived heaviness; $v$, perceived volume. Subscripts for these letters refer to the levels of the physical dimension or perceptual report ( + for the higher level, - for the lower level). 
Table 2

Marginal Response Invariance for Experiment 1

$\begin{array}{llc}M_{-} & p\left(m_{-} \mid M_{-} V_{-}\right) & .68 \\ & p\left(m_{-} \mid M_{-} V_{+}\right) & .89 \\ M_{+} & z & -14.55^{* *++} \\ & p\left(m_{+} \mid M_{+} V_{-}\right) & .81 \\ & p\left(m_{+} \mid M_{+} V_{+}\right) & .63 \\ V_{-} & p\left(v_{-} \mid M_{-} V_{-}\right) & 10.97 * *++ \\ & p\left(v_{-} \mid M_{+} V_{-}\right) & .90 \\ & z & .86 \\ V_{+} & p\left(v_{+} \mid M_{-} V_{+}\right) & 3.63^{* *} \\ & p\left(v_{+} \mid M_{+} V_{+}\right) & .88 \\ & z & .87 \\ \end{array}$

Note- $M$, mass; $V$, volume; $m$, perceived heaviness, $v$, perceived volume. Subscripts for these letters refer to the levels of the physical dimension or perceptual report ( + for the higher level, - for the lower level). $\quad * * p<$ .001 for pooled analyses. $\quad++p<.001$ for individual analyses (see the text).

mass decreased ( $p<.001$ ). Individually, however, only 10 out of 15 participants followed this trend, a number that did not reach significance $(z=1.69)$. The results from the same data for the large stimuli reveal that the proportion of large responses remained unchanged as mass increased. Individually, the sign test again showed no significant difference between these proportions ( 8 participants showed an increase, 5 showed a decrease, and 2 showed no difference; $z=.77$ ).

\section{Marginal Signal Detection Analyses}

Analyses of the pooled data for perceived heaviness showed that the $d$, value for perceived heaviness was significantly greater for the large stimuli than for the small stimuli ( $p<.001$; see Table 3 ). Individually, 13 out of 15 participants followed the same trend $(z=2.95, p<.01)$. This change in discriminability implies a lack of perceptual separability of volume and mass in relation to perceived heaviness. There were also significant variations in $C$ for perceived heaviness across levels of volume. Analyses of the pooled data for the small stimuli revealed that $C$ was negative, indicating that the participants were biased toward responding heavy, whereas for the large stimuli, $C$ was positive, indicating that they were biased toward responding light. Individually, 14 out of 15 participants followed the same trend $(z=3.48, p<.001)$. However, because marginal response invariance did not hold for perceived heaviness, no conclusions may be made about decisional separability on the basis of these analyses.

Analyses of the pooled data for perceived volume showed that the $d$ ' values for perceived volume did not differ significantly as mass increased (see Table 3 ). Individually, the sign test also showed no significant difference between these $d$ 'values ( 6 participants showed an increase, and 9 showed a decrease; $z=1.43$ ). This invariance in discriminability suggests that volume and mass are probably perceptually separable in relation to perceived size. Regarding decisional bias, analyses of the pooled data revealed that $C$ was significantly greater for the light stimuli than for the heavy stimuli $(p<.001)$. Individually, 12 out of 15 participants followed the same trend, a number that closely approached significance with the sign test $(z=$ $2.46, p=.0139$ ). These results suggest a lack of decisional separability in relation to perceived volume.

\section{Sampling Independence}

For each stimulus, the proportion of correct joint responses was compared with the product of the proportions of correct responses on each dimension. Analyses of the pooled data showed that none of these differences was significant (see Table 4). Individually, however, the difference for the small and light stimulus did reach significance at the $p<.01$ level with the sign test $(z=2.95$; for 13 participants, the actual joint proportion was smaller than the predicted one). For the remaining three stimuli, the differences failed to reach significance with the sign test. [For the large and light stimulus, the actual joint proportion was smaller for 5 participants, greater for 9 , and equal for $1(z=1.03)$; for the small and heavy stimulus, the actual joint proportion was smaller for 3 participants, greater for 11 , and equal for $1(z=2.07)$; and for the large and heavy stimulus, the actual joint proportion was smaller for 9 participants, greater for 5 , and equal for 1 $(z=1.03)$.] These results imply that volume and mass may be perceptually independent in relation to both perceived heaviness and perceived volume.

\section{Conditional Signal Detection Analyses}

Analyses of the pooled data for perceived heaviness at both levels of physical volume showed that $d$ 'values did not differ significantly as reports of perceived volume changed from small to large (see Table 5). Individually, the sign test also showed no significant difference between these $d$ ' values. [For the small stimuli, 8 participants showed an increase, and 7 showed a decrease $(z=1.29)$; for the large stimuli, 10 showed an increase, and 5 showed a decrease $(z=1.69)$.] Regarding decisional bias, analyses of the pooled data at both levels of physical volume revealed that $C$ was significantly greater when the participants reported that the stimuli were large, as compared

Table 3

Marginal Signal Detection Analyses for Experiment 1

\begin{tabular}{|c|c|c|c|c|}
\hline & $d$ & $S E_{d}^{2}$ & $C$ & $S E_{C}^{2}$ \\
\hline \multicolumn{5}{|c|}{ Perceived Heaviness } \\
\hline $\begin{array}{l}V_{-} \\
V_{+} \\
z\end{array}$ & $\begin{array}{l}1.35 \\
1.59 \\
-3.32 * *+\end{array}$ & $\begin{array}{l}0.003 \\
0.003\end{array}$ & $\begin{array}{c}-0.21 \\
0.46 \\
-18.05 * *++\end{array}$ & $\begin{array}{l}0.001 \\
0.001\end{array}$ \\
\hline \multicolumn{5}{|c|}{ Perceived Volume } \\
\hline $\begin{array}{l}M_{-} \\
M_{+} \\
z\end{array}$ & $\begin{array}{l}2.47 \\
2.27 \\
2.27\end{array}$ & $\begin{array}{l}0.004 \\
0.003\end{array}$ & $\begin{array}{c}0.05 \\
-0.07 \\
2.84 *\end{array}$ & $\begin{array}{l}0.001 \\
0.001\end{array}$ \\
\hline
\end{tabular}

Note- $M$, mass; $V$, volume. Subscripts for these letters refer to the level of the physical dimension (+ for the higher level, - for the lower level). $* p<.01$ for pooled analyses. $* * p<.001$ for pooled analyses. $\quad+p<.01$ for individual analyses (see the text). $\quad++p<.001$ for individual analyses (see the text). 
Table 4

Test of Sampling Independence for Experiment 1

\begin{tabular}{llr}
$M_{-} V_{-}$ & $p\left(m_{-} v_{-}\right)$ & .59 \\
& $p\left(m_{-}\right) * p\left(v_{-}\right)$ & .61 \\
$M_{-} V_{+}$ & $z$ & $-1.06+$ \\
& $p\left(m_{-} v_{+}\right)$ & .81 \\
& $p\left(m_{-}\right) * p\left(v_{+}\right)$ & .79 \\
$M_{+} V_{-}$ & $z$ & 1.15 \\
& $p\left(m_{+} v_{-}\right)$ & .71 \\
$M_{+} V_{+}$ & $p\left(m_{+}\right) * p\left(v_{-}\right)$ & .70 \\
& $z$ & 1.11 \\
& $p\left(m_{+} v_{+}\right)$ & .55 \\
& $p\left(m_{+}\right) * p\left(v_{+}\right)$ & .56 \\
& $z$ & -0.67 \\
\hline
\end{tabular}

Note- $M$, mass; $V$, volume; $m$, perceived heaviness; $v$, perceived volume. Subscripts for these letters refer to the levels of the physical dimension or perceptual report ( + for the higher level, - for the lower level). $\quad+p<.01$ for individual analyses (see the text).

with when they reported that they were $\operatorname{small}(p<.001)$. The participants were increasingly biased toward reporting light when they reported that the stimuli were large, regardless of whether they actually were large. Individually, for the small stimuli, 13 out of 15 participants followed the same trend $(z=2.95, p<.01)$. For the large stimuli, although only approaching significance, 12 out of 15 followed the same trend $(z=2.46, p=.0139)$. These analyses imply a lack of decisional separability of volume and mass in relation to perceived heaviness.

Analyses of the pooled data for perceived volume at both levels of mass showed that $d$ values did not differ significantly as reports of perceived heaviness changed from light to heavy (see Table 5). Individually, the sign test also showed no significant difference between these $d$. values. [For the light stimuli, 3 participants showed an increase, and 12 showed a decrease $(z=2.46)$; for the heavy stimuli, 10 showed an increase, and 5 showed a decrease $(z=1.69)$.] Regarding decisional bias, analyses of the pooled data for both levels of mass revealed that $C$ was significantly greater when participants reported that the stimuli were heavy, as compared with when they reported that they were light $(p<.001)$. The participants were increasingly biased toward reporting small when they thought the stimuli were heavy, regardless of whether they actually were heavy. For the light stimuli, 14 out of 15 participants followed the same trend $(z=3.84, p<.001)$. For the heavy stimuli, although only approaching significance, 11 out of 15 followed the same trend $(z=2.04, p<$ $.05)$. The results of these analyses provide further support for a lack of decisional separability of volume and mass in relation to perceived volume.

\section{Discussion}

On the basis of both the pooled and the individual results from all the analyses, a lack of both perceptual and decisional separability may be considered to be the source of the size-weight illusion when haptic touch is used but visual input is not allowed. A lack of perceptual separability suggests that mass and volume exist as a combined stimulus property that is detected and used in the perception of heaviness. The conclusion that the size-weight illusion may result from a lack of perceptual separability has been made previously for dynamic touch (Amazeen, 1999). ${ }^{3}$ For dynamic touch, it was concluded that mass and volume were combined through their joint influence on the object's rotational inertia, a property that has been shown to govern perceived heaviness by dynamic touch (Amazeen, 1997, 1999; Amazeen \& Turvey, 1996; Kloos \& Amazeen, 2002). The present data do not allow us to make any determination regarding the particular combination of mass and volume that is suggested by the lack of perceptual separability. Nevertheless, the lack of perceptual separability in the present experiment may be the result of haptic perception's relying on rotational inertia in the same way that dynamic touch does.

The present findings are in contrast with those from dynamic touch, in which volume and mass were decisionally

Table 5

Conditional Signal Detection Analyses for Experiment 1

\begin{tabular}{|c|c|c|c|c|c|c|c|c|}
\hline \multicolumn{9}{|c|}{ Perceived Heaviness } \\
\hline & \multicolumn{4}{|c|}{$V_{-}$} & \multicolumn{4}{|c|}{$V_{+}$} \\
\hline & $d$ & $S E_{d}^{2}$ & $C$ & $\overline{S E_{C}^{2}}$ & $d$ & $S E_{d}^{2}$ & $C$ & $S E_{C}^{2}$ \\
\hline$\overline{v_{-}}$ & 1.37 & 0.003 & -0.29 & 0.001 & 1.33 & 0.021 & 0.02 & 0.005 \\
\hline$v_{+}$ & 1.59 & 0.024 & 0.32 & 0.006 & 1.67 & 0.004 & 0.53 & 0.001 \\
\hline$z^{\top}$ & -1.31 & & $-7.25^{* *+}+$ & & -2.17 & & $-6.52 * *$ & \\
\hline \multicolumn{9}{|c|}{ Perceived Volume } \\
\hline & \multicolumn{4}{|c|}{$M_{-}$} & \multicolumn{4}{|c|}{$M_{+}$} \\
\hline & $d$ & $S E_{d}^{2}$ & $C$ & $S E_{C}^{2}$ & $d$ & $S E_{d}^{2}$ & $C$ & $S E_{C}^{2}$ \\
\hline$\overline{m_{-}}$ & 2.42 & 0.005 & -0.08 & 0.001 & 2.10 & 0.013 & -0.35 & $\overline{0.003}$ \\
\hline$m_{+}$ & 2.32 & 0.022 & 0.57 & 0.005 & 2.29 & 0.005 & 0.03 & 0.001 \\
\hline$z^{+}$ & 0.60 & & $-8.00 * *++$ & & -1.37 & & $-5.76^{* *}$ & \\
\hline
\end{tabular}

Note- $M$, mass; $V$, volume; $m$, perceived heaviness; $v$, perceived volume. Subscripts for these letters refer to the levels of the physical dimension or perceptual report ( + for the higher level, - for the lower level). $* * p<.001$ for pooled analyses. $+p<.01$ for individual analyses (see the text). $\quad++p<.001$ for individual analyses (see the text). 
separable. The lack of decisional separability in the present experiment indicates that the placement of the decision criterion for perceived heaviness is a function of both mass and volume. The participants were biased toward responding light for the large stimuli and heavy for the small stimuli. This response bias seems to exist when participants have access to additional volumetric information through haptic touch, as compared with the purely dynamic touch scenario of Amazeen (1999). The existence of this additional decisional effect may explain why other studies, in which the strengths of the size-weight illusion with different modes of touch were compared, have shown that the effects of volume on the report of perceived heaviness are strongest through haptic touch (Ellis \& Lederman, 1993; Pick \& Pick, 1967). When haptic touch is used without visual input, the participant's report of perceived heaviness may be affected by volume through volume's influence on rotational inertia and through volume's influence on the decision criterion. These two separate effects may combine to produce a strong effect of volume on the report of perceived heaviness.

In relation to perceived volume, similar to the dynamic touch study (Amazeen, 1999), volume and mass appeared to be both perceptually independent and perceptually separable (i.e., perceived volume was most likely a function of volume alone). Unlike the dynamic touch study, however, a lack of decisional separability was found in the present experiment. The placement of one's decision criterion for perceived volume appears to be a function of both volume and mass when haptic touch is used and visual input is not allowed.

\section{EXPERIMENT 2}

A number of studies have investigated the role of vision in the size-weight illusion. When comparisons are made between the effects of volume through haptic touch and through vision, it appears as though the illusion is strongest with haptic information about volume (Ellis \& Lederman, 1993; Masin \& Crestoni, 1988; Pick \& Pick, 1967). That is not to say, however, that visual information about volume is unimportant. The aforementioned studies in which vision and haptic touch were compared have shown that visual information about volume is sufficient on its own to produce the illusion. Furthermore, manipulations of vision alone, using either concave or convex lenses, are also sufficient to produce a size-weight illusion (Koseleff, 1957). And in research on dynamic touch, the effects of vision on the report of perceived heaviness appeared to superimpose on the effects of dynamic touch (Amazeen, 1997). The qualitatively different results of these studies warrant continued research on the role of vision in the perception of heaviness. To determine the effects of visual input on the independence and separability of volume and mass, Experiment 2 used a method identical to that in Experiment 1 , with the only exception being that the participants could view the stimuli as they held them.

\section{Method}

\section{Participants}

Ten female and 5 male Arizona State University undergraduate students participated in this experiment in partial fulfillment of an introductory psychology course requirement. Eleven participants were right-handed (based on self-reports of which hand they throw a ball with and write with), and 4 participants were left-handed. All the participants were naive as to the purpose of this study.

\section{Materials}

The stimuli used in this experiment were the same wooden cylinders as those used in Experiment 1.

\section{Procedure}

The procedure for this experiment was almost identical to that in Experiment 1, with the exception that the participant was allowed to view the stimulus in his or her hand during each trial. During the trials, all other stimuli were hidden from view by the curtain.

\section{Results}

The response frequencies for all stimuli and all participants combined are presented in Table 6. Collapsing across levels of mass, as physical volume increased, the frequency of light responses increased (1,234 for the small stimuli and 1,864 for the large stimuli), and the frequency of heavy responses decreased (1,766 for the small stimuli and 1,136 for the large stimuli). These data display a size-weight illusion, consistent with the results of nonGRT researchers (e.g., Stevens \& Rubin, 1970). As in Experiment 1 , analyses were conducted on the pooled data and on a participant-by-participant basis via the sign test. Test failures were suggested when significant differences in values (e.g., $d$ ' for perceived heaviness across levels of volume) occurred for both types of analyses. To correct for alpha inflation, an alpha level of .01 was again used for all the analyses.

\section{Marginal Response Invariance}

The results from the pooled data for perceived heaviness reveal that, for both levels of mass, the proportion of light responses increased significantly as volume increased $(p<.001$; see Table 7$)$. For each level of mass, all 15 participants followed the same trend $(p<.001)$. The results from the pooled data for perceived volume reveal that the proportions of correct responses were invariant as mass increased. The sign test also showed no significant difference between these proportions when calculated for

Table 6

Response Frequencies for Experiment 2

\begin{tabular}{lrrrr}
\hline Report & $M_{-} V_{-}$ & $M_{-} V_{+}$ & $M_{+} V_{-}$ & $M_{+} V_{+}$ \\
\hline$m_{-} v_{-}$ & 1,003 & 51 & 118 & 22 \\
$m_{-} v_{+}$ & 75 & 1,351 & 38 & 440 \\
$m_{+} v_{-}$ & 407 & 11 & 1,263 & 65 \\
$m_{+} v_{+}$ & 15 & 87 & 81 & 973 \\
\hline
\end{tabular}

Note- $M$, mass; $V$, volume; $m$, perceived heaviness; $v$, perceived volume. Subscripts for these letters refer to the levels of the physical dimension or perceptual report ( + for the higher level, - for the lower level). 
Table 7

Marginal Response Invariance for Experiment 2

$\begin{array}{llc}M_{-} & p\left(m_{-} \mid M_{-} V_{-}\right) & .72 \\ & p\left(m_{-} \mid M_{-} V_{+}\right) & .93 \\ M_{+} & z & -15.63^{* *++} \\ & p\left(m_{+} \mid M_{+} V_{-}\right) & .90 \\ & p\left(m_{+} \mid M_{+} V_{+}\right) & .69 \\ V_{-} & z & 13.81^{* *++} \\ & p\left(v_{-} \mid M_{-} V_{-}\right) & .94 \\ & z\left(v_{-} \mid M_{+} V_{-}\right) & .92 \\ V_{+} & p\left(v_{+} \mid M_{-} V_{+}\right) & 2.08 \\ & p\left(v_{+} \mid M_{+} V_{+}\right) & .96 \\ & z & .94 \\ \end{array}$

Note- $M$, mass; $V$, volume; $m$, perceived heaviness; $v$, perceived volume. Subscripts for these letters refer to the levels of the physical dimension or perceptual report ( + for the higher level, - for the lower level). $* * p<.001$ for pooled analyses. $\quad++p<.001$ for individual analyses (see the text).

each individual participant. [For the small stimuli, 2 participants showed an increase, 9 participants showed a decrease, and 4 showed no difference $(z=1.81)$; for the large stimuli, 3 showed an increase, 9 showed a decrease, and 3 showed no difference $(z=1.55)$.]

\section{Marginal Signal Detection Analyses}

Analyses of the pooled data for perceived heaviness showed that the $d$ ' values were invariant as volume increased (see Table 8). Individually, the sign test also showed no significant difference between these $d$ values when calculated for each individual participant (10 showed an increase, 4 showed a decrease, and 1 showed no difference; $z=1.55$ ). This invariance in discriminability implies that volume and mass are probably perceptually separable in relation to perceived heaviness. This contrasts with the findings from Experiment 1, in which haptic touch without vision was used, and from Amazeen (1999), in which dynamic touch without vision was used. In each of those experiments, there was a lack of perceptual separability of volume and mass. With respect to bias, there were significant variations in $C$ for perceived heaviness across levels of volume $(p<.001)$. Analyses of the pooled data for the small stimuli revealed that $C$ was negative, indicating that the participants were biased toward responding heavy, whereas for the large stimuli, $C$ was positive, indicating that they were biased toward responding light. All 15 participants followed the same trend $(p<$ .001 ). These results suggest a lack of decisional separability of volume and mass in relation to perceived heaviness.

Analyses of the pooled data for perceived volume showed that both $d^{\prime}$ and $C$ were invariant as mass increased (see Table 8). Individually, the sign test also showed no significant difference between these $d$ and $C$ values. [Regarding $d$ ' comparisons, 3 participants showed an increase, 11 showed a decrease, and 1 showed no difference $(z=2.07)$; regarding $C$ comparisons, 5 showed an increase, 9 showed a decrease, and 1 showed no difference $(z=1.03)$.] This invariance in discriminability and deci- sional bias implies that volume and mass are probably both perceptually and decisionally separable in relation to perceived size.

\section{Sampling Independence}

For each stimulus, the proportion of correct joint responses was compared with the product of the proportions of correct responses on each dimension. Analyses of the pooled data showed that none of these pairs was significantly different (see Table 9). Individually, none of the comparisons was significant with the sign test. [For the small and light stimulus, the actual joint proportion was smaller than the predicted one for 10 participants, equal for 4 , and greater for $1(z=-2.32)$; for the large and light stimulus, the actual joint proportion was smaller than the predicted one for 6 participants, equal for 1, and greater for $8(z=0.52)$; for the small and heavy stimulus, the actual joint proportion was smaller than the predicted one for 3 participants, equal for 3 , and greater for $9(z=1.55)$; and for the large and heavy stimulus, the actual joint proportion was smaller than predicted for 10 participants, equal for 1 , and greater for $4(z=-1.03)$.] Overall, these results, similar to those in the haptic touch without visual input experiment, imply that volume and mass may be perceptually independent in relation to both perceived heaviness and perceived size.

\section{Conditional Signal Detection Analyses}

Analyses of the pooled data for perceived heaviness at both levels of physical volume showed that $d$ 'values did not differ significantly as reports of perceived volume changed from small to large (see Table 10). Individually, the sign test also showed no significant difference in these $d$ pairs. [For the small stimuli, 4 participants showed an increase, and 11 showed a decrease $(z=2.04)$; for the large stimuli, 10 showed an increase, and 5 showed a decrease $(z=1.69)$.] Regarding decisional bias, analyses of the pooled data at both levels of physical volume revealed that $C$ was significantly greater when the participants reported that the stimuli were large, as compared with when they reported that they were small $(p<.01)$. The participants were increasingly biased toward reporting light

Table 8

Marginal Signal Detection Analyses for Experiment 2

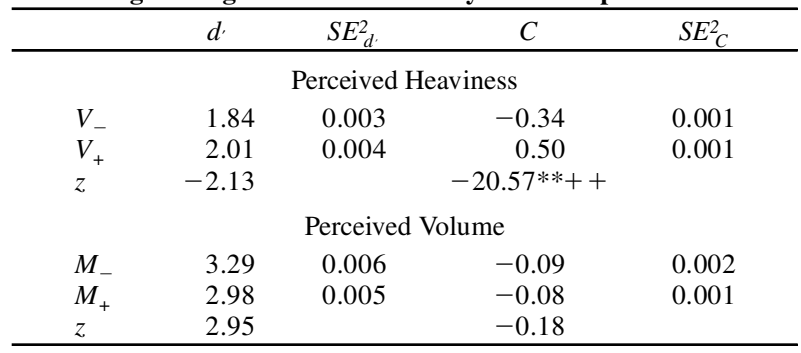

Note- $M$, mass; $V$, volume. Subscripts for these letters refer to the level of the physical dimension ( + for the higher level, - for the lower level). ${ }^{* *} p<.001$ for pooled analyses. $\quad++p<.001$ for individual analyses (see the text). 
Table 9

Test of Sampling Independence for Experiment 2

$\begin{array}{llr}M_{-} V_{-} & p\left(m_{-} v_{-}\right) & .67 \\ & p\left(m_{-}\right) * p\left(v_{-}\right) & .68 \\ M_{-} V_{+} & z & -0.40 \\ & p\left(m_{-} v_{+}\right) & .90 \\ & p\left(m_{-}\right) * p\left(v_{+}\right) & .90 \\ M_{+} V_{-} & p\left(m_{+} v_{-}\right) & 0.42 \\ & p\left(m_{+}\right) * p\left(v_{-}\right) & .84 \\ & z & .83 \\ M_{+} V_{+} & p\left(m_{+} v_{+}\right) & 1.26 \\ & p\left(m_{+}\right) * p\left(v_{+}\right) & .64 \\ & z & .65 \\ & & -0.18\end{array}$

Note- $M$, mass; $V$, volume; $m$, perceived heaviness; $v$, perceived volume. Subscripts for these letters refer to the levels of the physical dimension or perceptual report ( + for the higher level, - for the lower level).

when they thought the stimuli were large, regardless of whether they actually were large. Individually, 13 out of 15 participants followed the same trend for the small stimuli $(z=2.95, p<.01)$, and 14 out of 15 participants followed the same trend for the large stimuli $(z=3.84, p<$ $.001)$. These results suggest a lack of decisional separability of volume and mass in relation to perceived heaviness.

Analyses of the pooled data for perceived volume showed that for the light stimuli, $d$ ' did not differ significantly as reports of perceived heaviness increased from light to heavy (see Table 10). Individually, the sign test also showed no significant difference between these $d$. values ( 5 participants showed an increase, and 10 showed a decrease; $z=1.69$ ). Analyses of the pooled data for the heavy stimuli, however, revealed that $d$ ' was significantly greater when the participants reported that the stimuli were heavy, as compared with when they reported that they were light. Individually, 13 out of 15 participants followed the same trend $(z=2.95, p<.01)$. Regarding decisional bias, analyses of the pooled data for both levels of mass revealed that $C$ was significantly greater when the participants reported that the stimuli were heavy, as compared with when they reported that they were light $(p<$ .001). The participants were increasingly biased toward reporting small when they thought the stimuli were heavy, regardless of whether they actually were heavy. Individually, 14 out of 15 participants followed the same trend for both levels of mass $(z=3.84, p<.001)$. These results imply a lack of decisional separability of volume and mass in relation to perceived volume.

\section{Discussion}

A comparison of the response frequency distributions from Experiments 1 and 2 (see Tables 1 and 6) suggests that the participants were overall more accurate when vision was allowed. It is not surprising that the participants would be more accurate at discriminating volume in Experiment 2. It appears, though, that the participants may have also been more accurate at discriminating mass when vision was allowed in Experiment 2. One way of assessing this effect is by comparing the discriminability of mass in each experiment. The addition of vision in Experiment 2 led to significantly greater discrimination of mass with vi$\operatorname{sion}\left(d^{\prime}=1.76, S E_{d}=0.001\right)$, as compared with without vi$\operatorname{sion}\left(d^{\prime}=1.38, S E_{d}=0.001 ; z=-7.34, p<.001\right)$.

There were two main findings in Experiment 2. The first was that the size-weight illusion with both haptic touch and visual input was associated with a lack of decisional separability. This contrasts with the haptic touch only experiment, in which there was a lack of perceptual separability of volume and mass (in addition to the lack of decisional separability). When vision was allowed in Experiment 2, physical volume influenced the report of perceived heaviness, but most likely not the perceptual effect itself. This may be related to the second main finding, which was the increased discrimination of mass in the presence of visual information. Vision made the variations in mass more salient. This effect may be the result of vision's being used to separate the effects of mass and volume in perceived heaviness.

Table 10

Conditional Signal Detection Analyses for Experiment 2

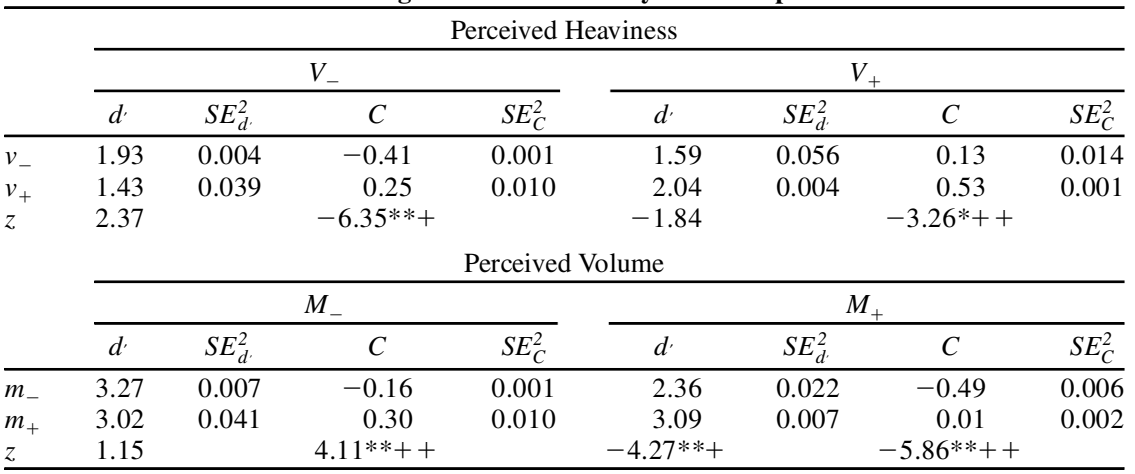

Note- $M$, mass; $V$, volume; $m$, perceived heaviness; $v$, perceived volume. Subscripts for these letters refer to the levels of the physical dimension or perceptual report $(+$ for the higher level, - for the lower level). $\quad * p<.01$ for pooled analyses. $\quad * * p<.001$ for pooled analyses. $\quad+p<.01$ for individual analyses (see the text). $\quad++p<.001$ for individual analyses (see the text). 
In relation to perceived volume, as in the haptic touch without visual input experiment, volume and mass were probably both perceptually independent and perceptually separable, but there was a lack of decisional separability of volume and mass, on the basis of the signal detection analyses. The placement of one's decision criterion for perceived size appears to be a function of both volume and mass.

\section{GENERAL DISCUSSION}

The present experiments applied a multidimensional signal detection analysis to the size-weight illusion in order to examine perceptual separability, perceptual independence, and decisional separability of volume and mass. Use of this analysis required a feature-complete factorial design, in which there was a factorial combination of two levels of both volume and mass and a perceptual report for each of these dimensions was given. In keeping with signal detection experiments, the pair members selected for the two dimensions were not physically very different from one another. Although this stimulus set was sparse, the data patterns from both experiments are consistent with those of researchers who used a number of different parameter values (e.g., Ellis \& Lederman, 1993; Stevens \& Rubin, 1970), providing additional support for the validity of this analysis that allows for an indirect test of the validity of the three corresponding types of illusion models. The findings reveal that different models may be appropriate, depending on the type of touch and whether visual input was allowed (see Table 11).

\section{Appropriate Classes of Models}

One class of size-weight illusion models suggests that the illusion is due to percept-percept coupling, or an apparent lack of perceptual independence (e.g., Anderson, 1970, 1972). Such models assume that the initial perception of heaviness is a function of mass only and that the initial perception of volume is a function of volume only. With these assumptions in mind, these models claim that the influence of physical volume on report of perceived heaviness is due to an interaction of these two initial perceptual effects. However, the results of both experiments, along with those of Amazeen (1999), imply that volume and mass may be perceptually independent and that the illusion does not result from percept-percept coupling.

\section{Table 11}

Summary of Findings on Perceptual Independence (PI), Perceptual Separability (PS), and Decisional Separability (DS)

PI PS DS

\section{Perceived Heaviness}

Amazeen (1999)

Experiment 1

Experiment 2

\section{yes}

yes

yes

\section{no}

no

yes

yes

Perceived Volume

Amazeen (1999)

Experiment 1

Experiment 2

$\begin{array}{lll}\text { yes } & \text { yes } & \text { yes } \\ \text { yes } & \text { yes } & \text { no } \\ \text { yes } & \text { yes } & \text { no }\end{array}$

The second class of illusion models claims a lack of perceptual separability of volume and mass. Models claiming that the illusion is due to a lack of perceptual separability suggest that perceived heaviness is a function of both mass and volume. For example, according to the density model (H. E. Ross, 1969; J. Ross \& Di Lollo, 1970), perceived heaviness is a function of density, rather than of mass alone. Another model claiming that the illusion is due to a lack of perceptual separability is the inertial model (Amazeen, 1999; Amazeen \& Turvey, 1996), which claims that perceived heaviness is a function of an object's inertial properties (mass and rotational inertia) that mass and volume are inseparable components of perceived heaviness because they are inseparable components of rotational inertia. Results from Experiment 1 revealed that, when haptic touch is used in the absence of visual input, volume and mass are not perceptually separable. Perceived heaviness is dependent on the level of physical volume. Amazeen (1999) also found a lack of perceptual separability, using dynamic touch, and related that effect to the role of rotational inertia. Because the participants in Experiment 1 still had access to rotational inertia through the muscular dynamics of lifting and holding, the lack of perceptual separability in this experiment may have also been the result of participants' relying on rotational inertia to perceive heaviness, although that particular model was not tested in the present experiments.

The results of both experiments call for an effect of volume on the decision criterion for perceived heaviness in models of the size-weight illusion by haptic touch with and without vision. Existing models of the size-weight illusion have not addressed explicitly such a lack of decisional separability. It may be that the effects of expected heaviness that are often treated as influencing the perceptual effect (e.g., Anderson, 1970, 1972; Davis \& Roberts, 1976; H. E. Ross \& Gregory, 1970) may, in fact, be influencing the decision criterion instead. Treating expectations as affecting the decision criterion, rather than the perceptual effects, is consistent with signal detection theory. When participants in a signal + noise identification task are given different expectations regarding the likelihood of a signal's being presented, their responses lie along a single receiver-operating characteristic curve, indicating that the perceptual effect does not change but that the decision criterion does (Macmillan \& Creelman, 1991). Similarly, given two objects of the same mass but differing in volume, one may have an expectation that the larger object should be heavier than the smaller object. When both are wielded, however, the failure to meet this expectation may bias one to report that the larger object feels lighter than the smaller object.

\section{Effects of Vision}

Consistent with previous research in which the effects of haptic and visual information about volume were compared (Ellis \& Lederman, 1993; Pick \& Pick, 1967), a size-weight illusion was demonstrated in each experiment. That is, increasing physical volume produced a decrease in the magnitude of reports of perceived heaviness. 
However, also consistent with those same studies, the effects of haptic and visual information were not identical in the present experiments. Previous research has suggested that the illusion is strongest with haptic information about volume. By identifying the source of the illusion, the present analysis extends those conclusions.

When the participants had only haptic contact with the object (i.e., it is held but not viewed), there was an effect of physical volume on perceived heaviness through a lack of perceptual separability and an effect of volume on the decision criterion through a lack of decisional separability. The combined result of these two effects would produce a robust illusion in this modality. When the participants were allowed to view the object, however, the effect of volume on the perceptual effect for perceived heaviness seemed to disappear, leaving only an effect on the decision criterion. These qualitative differences between modalities may be related to the quantitative differences reported in previous studies. Both Ellis and Lederman (1993) and Pick and Pick (1967) showed that the strength of the illusion in the haptic + vision case was equal to, or marginally less than, the strength of the illusion in the purely haptic case. If the same effects as those found in the present experiments were operating in those previous studies, it is possible that visual input leads to more perceptual separability of these dimensions and thereby reduces the illusion. More precise predictions regarding the strength of the illusion, however, would require more information about the relative strengths of the decisional effect in each case.

The effects of vision were not limited to the influence of volume on perceived heaviness. When the participant was able to view the object in his or her hand, he or she was also more sensitive to variations in mass. It is unlikely, then, that vision was simply providing access to the object's geometric properties. Rather, vision appears to provide information about the object's dynamic properties and may do so through the perception of the object's motions. Such a conclusion could also be derived from the results of Masin and Crestoni (1988). Masin and Crestoni demonstrated that vision alone was sufficient to produce the size-weight illusion, but only when participants could view the stimulus during the lift. The illusion disappeared when participants could view the stimulus before but not during the lift. Apparently, vision was used to monitor the object's motions (or perhaps the kinematics of the hand movements), rather than its size alone. The hypothesis that optical kinematics can specify mass-based properties has received experimental support (e.g., Runeson, 1977; Runeson \& Frykholm, 1981, 1983). However, the particular patterns of motion that are detected by vision and used to establish the decision criterion would still need to be determined.

\section{Conclusion}

As one goes from dynamic touch (kinesthetic stimulation) to haptic touch (kinesthetic and tactile stimulation) to haptic touch with visual input (kinesthetic, tactile, and visual stimulation), volume and mass seem to become more perceptually separable and less decisionally separable. The implication of these findings is that models of the size-weight illusion must be sensitive to the modality of perception being used and must address both perceptual and decisional effects. The connection between these and previous results suggests that the perceptual effect may be the result of perceived heaviness's being a function of rotational inertia in instances of dynamic touch and haptic touch without vision and that the decisional effect may be the result of an expectation regarding the object's motions when lifted in instances of haptic touch with and without vision.

\section{REFERENCES}

Amazeen, E. L. (1997). The effects of volume on perceived heaviness by dynamic touch: With and without vision. Ecological Psychology, 9, 245-263.

Amazeen, E. L. (1999). Perceptual independence of size and weight by dynamic touch. Journal of Experimental Psychology: Human Perception \& Performance, 25, 102-119.

Amazeen, E. L., \& Turvey, M. T. (1996). Weight perception and the haptic size-weight illusion are functions of the inertia tensor. Journal of Experimental Psychology: Human Perception \& Performance, 22, 213-232.

Anderson, N. H. (1970). Averaging model applied to the size-weight illusion. Perception \& Psychophysics, 8, 1-4.

Anderson, N. H. (1972). Cross-task validation of functional measurement. Perception \& Psychophysics, 12, 389-395.

Ashby, F. G., \& Townsend, J. T. (1986). Varieties of perceptual independence. Psychological Review, 93, 154-179.

Charpentier, A. (1891). Analyse experimentale de quelques elements de la sensation de poids [Experimental study of some aspects of weight perception]. Archives de Physiologie Normales et Pathologiques, 3, 122-135.

Davis, C. M., \& Roberts, W. (1976). Lifting movements in the size-weight illusion. Perception \& Psychophysics, 20, 33-36.

Ellis, R. R., \& Lederman, S. J. (1993). The role of haptic versus visual volume cues in the size-weight illusion. Perception \& Psychophysics, 53, 315-324.

Epstein, W. (1982). Percept-percept couplings. Perception, 11, 75-83. Gibson, J. J. (1966). The senses considered as perceptual systems. Boston: Houghton Mifflin.

Gourevitch, V., \& Galanter, E. (1967). A significance test for one parameter isosensitivity functions. Psychometrika, 32, 25-33.

Green, D. M., \& Swets, J. A. (1966). Signal detection theory and psychophysics. New York: Wiley.

Hautus, M. J. (1995). Corrections for extreme proportions and their biasing effects on estimated values of $d$. Behavior Research Methods, Instruments, \& Computers, 27, 46-51.

Jones, L. A. (1986). Perception of force and weight: Theory and research. Psychological Bulletin, 100, 29-42.

KADLEC, H. (1995). Multidimensional signal detection analyses (MSDA) for testing separability and independence: A Pascal program. Behavior Research Methods, Instruments, \& Computers, 27, 442-458.

KADLEC, H. (1999a). MSDA_2: Updated version of software for multidimensional signal detection analyses. Behavior Research Methods, Instruments, \& Computers, 31, 384-385.

KadLec, H. (1999b). Statistical properties of $d^{\prime}$ and $\beta$ estimates of signal detection theory. Psychological Methods, 4, 22-43.

KADLEC, H., \& Hicks, C. L. (1998). Invariance of perceptual spaces and perceptual separability of stimulus dimensions. Journal of Experimental Psychology: Human Perception \& Performance, 24, 80-104.

Kadlec, H., \& Townsend, J. T. (1992a). Implications of marginal and conditional detection parameters for the separabilities and independence of perceptual dimensions. Journal of Mathematical Psychology, 36, 325-374. 
Kadlec, H., \& Townsend, J. T. (1992b). Signal detection analyses of dimensional interactions. In F. G. Ashby (Ed.), Multidimensionalmodels of perception and cognition (pp. 181-227). Hillsdale, NJ: Erlbaum.

Kloos, H., \& Amazeen, E. L. (2002). Perceiving heaviness by dynamic touch: An investigation of the size-weight illusion in preschoolers. British Journal of Developmental Psychology, 20, 171-183.

Koseleff, P. (1957). Studies in the perception of heaviness. Acta Psychologica, 13, 242-252.

Loomis, J. M., \& Lederman, S. J. (1986). Tactual perception. In K. R. Boff, L. Kaufman, \& J. P. Thomas (Eds.), Handbook of perception and human performance (pp. 31.1-31.41). New York: Wiley.

Macmillan, N. A., \& Creelman, C. D. (1991). Detection theory: A user's guide. New York: Cambridge University Press.

Marascuilo, L. A. (1970). Extensions of the significance test for oneparameter signal detection hypotheses. Psychometrika, 35, 237-243.

Masin, S. C., \& Crestoni, L. (1988). Experimental demonstration of the sensory basis of the size-weight illusion. Perception \& Psychophysics, 44, 309-312.

Miller, J. (1996). The sampling distribution of $d$. Perception \& Psychophysics, 58, 65-72.

Murray, D. J., Ellis, R. R., Bandomir, C. A., \& Ross, H. E. (1999). Charpentier (1891) on the size-weight illusion. Perception \& Psychophysics, 61, 1681-1685.

Pick, H. L., \& Pick, A. D. (1967). A developmental and analytic study of the size-weight illusion. Journal of Experimental Child Psychology, 5, 362-371.

Robertson, C. C., \& Morgan, B. J. T. (1990). Aspects of the design and analysis of signal detection experiments. British Journal of Mathematical \& Statistical Psychology, 42, 7-14.

Ross, H. E. (1969). When is a weight not illusory? Quarterly Journal of Experimental Psychology, 21, 346-355.

Ross, H. E., \& Gregory, R. L. (1970). Weight illusions and weight discrimination: A revised hypothesis. Quarterly Journal of Experimental Psychology, 22, 318-328.

Ross, J., \& Di Lollo, V. (1970). Differences in heaviness in relation to density and weight. Perception \& Psychophysics, 7, 161-162.

RUNESON, S. (1977). On the visual perception of dynamic events. Uppsala: University of Uppsala.

Runeson, S., \& FryKholm, G. (1981). Visual perception of lifted weight. Journal of Experimental Psychology: Human Perception \& Performance, 7, 733-740.

Runeson, S., \& Fry Kholm, G. (1983). Kinematic specification of dynamics as an informational basis for person and action perception: Expectation, gender recognition, and deceptive intention. Journal of Experimental Psychology: General, 112, 585-615.

Stevens, J. C., \& Rubin, L. L. (1970). Psychophysical scales of apparent heaviness and the size-weight illusion. Perception \& Psychophysics, 8, 225-230.
Thomas, R. D. (2001). Characterizing perceptual interactions in face identification using multidimensional signal detection theory. In M. J. Wenger \& J. T. Townsend (Eds.), Computational, geometric, and process perspectives on facial cognition: Contests and challenges (pp. 193-228). Mahwah, NJ: Erlbaum.

Wenger, M. J., \& IngValson, E. M. (2002). A decisional component of holistic encoding. Journal of Experimental Psychology: Learning, Memory, \& Cognition, 28, 872-892.

Wickens, T. D., \& OlzaK, L. A. (1992). Three views of association in concurrent detection ratings. In F. G. Ashby (Ed.), Multidimensional models of perception and cognition (pp. 229-252). Hillsdale, NJ: Erlbaum.

\section{NOTES}

1. To be consistent with signal detection theory, it is necessary to distinguish the perceptual effect on each dimension from the report of that perception. Volume and mass will be used to refer to the two physical dimensions, perceived volume and perceived heaviness will be used to identify the perceptual effects associated with each dimension, and reports of perceived volume and perceived heaviness will be used to identify the verbal reports made by the participant.

2 . Throughout this paper, physical dimensions are represented by capital letters $(M=$ mass, $V=$ volume $)$, and the participants' perceptual reports are represented by lowercase letters ( $m=$ perceived heaviness, $v=$ perceived volume). Subscripts for these letters refer to the level of the physical dimension or perceptual report ( + for the higher level, - for the lower level).

3. The tests of marginal response invariance and sampling independence were reported by Amazeen (1999) for a similar experiment in which participants reported perceived heaviness and volume, using only dynamic touch. The criteria of both tests were met $(p=.05)$. To facilitate comparisons among the size-weight illusion by dynamic touch and the modes of touch used in the present article, the additional tests of the estimated signal detection parameters can also be applied to those data (Amazeen, 1999, Table 4). A log-linear rule transformation was used to correct for cells with a frequency of zero (Hautus, 1995). For the marginal signal detection analyses, there was a significant change in $d^{\prime}(3.86$ at $V_{-}, 2.7$ at $\left.V_{+} ; z=2.2\right)$, but not in $C\left(-0.11\right.$ at $V_{-},-0.09$ at $V_{+} ; z=$ $-0.55)$. For the conditional signal detection analyses, there was no significant change in $d^{\prime}\left(V_{-}, 3.53\right.$ at $v_{-}, 4.04$ at $v_{+}, z=-0.53 ; V_{+}, 2.75$ at $v_{-}, 2.82$ at $\left.v_{+}, z=-0.09\right)$ or $C\left(V_{-},-0.08\right.$ at $v_{-},-0.08$ at $v_{+}, z=-0.01$; $V_{+},-0.51$ at $v_{-}, 0.29$ at $\left.v_{+}, z=-1.79\right)$. The results of these tests further support the conclusion of Amazeen (1999) that the size-weight illusion by dynamic touch resulted from a lack of perceptual separability.

(Manuscript received May 22, 2002; revision accepted for publication February 13, 2003.) 